\title{
Recognising meningococcal disease in primary care: qualitative study of how general practitioners process clinical and contextual information
}

\author{
Stephen Granier, Penny Owen, Roisin Pill, Lionel Jacobson
}

Department of General Practice and Primary Care, King's College School of Medicine and Dentistry,

London SE5 9PJ Stephen Granier, research fellow

Llanedeyrn Health Centre, Cardiff

CF3 7PN

Penny Owen,

general practitioner

Department of

General Practice,

University of Wales

College of

Medicine, Cardiff

CF3 7PN

Roisin Pill,

professor

Lionel Jacobson,

research fellow

Correspondence to:

Dr Granier

BMJ 1998;316:276-9

\begin{abstract}
Objectives: To describe the presentation of meningococcal disease in primary care; to explore how general practitioners process clinical and contextual information in children with meningococcal disease; and to describe how this information affects management.

Design: Qualitative analysis of semistructured interviews.

Setting: General practices in South Glamorgan. Subjects: 26 general practitioners who between January 1994 and December 1996 admitted 31 children (under 16 years of age) in whom meningococcal disease was diagnosed.
\end{abstract}

Main outcome measures: Categories of clinical rules and techniques used by general practitioners in processing each case.

Results: 22 children had rashes; in 16 of them the rashes were non-blanching. When present, a haemorrhagic rash was the most important factor in the doctor's decision to admit a child. 22 children had clinical features not normally expected in children with acute self limiting illnesses-for example, lethargy, poor eye contact, altered mental states, pallor with a high temperature, and an abnormal cry. Contextual information, such as knowledge of parents' consultation patterns and their normal degree of anxiety, played an important part in the management decisions in 15 cases. Use of penicillin was associated with the certainty of diagnosis and the presence and type of haemorrhagic rash.

Conclusion: The key clinical feature of meningococcal disease-a haemorrhagic rash-was present in only half of the study children. The general practitioners specifically hunted for the rash in some ill children, but doctors should not be deterred from diagnosing meningococcal disease and starting antibiotic treatment if the child is otherwise well, if the rash has an unusual or scanty distribution, or if the rash is non-haemorrhagic.

\section{Introduction}

Current knowledge on the presentation of meningococcal disease has been defined largely from the results of studies based in hospitals, where the disease is seen at a late stage. $^{1-8}$ General practitioners encounter meningococcal disease earlier, when signs and symptoms are often non-specific, making diagnosis difficult. Early treatment for meningococcal disease has been widely recommended and shown to improve outcome $^{7-11}$ in all but one study. ${ }^{12}$ Prompt treatment requires early recognition, but in up to a quarter of cases the diagnosis is delayed by more than 48 hours after the onset of the illness. ${ }^{1-13}$

A review of the published research on meningococcal disease found only one study on the recognition of meningococcal disease (predominantly meningitis of all types) using data from primary care.$^{14}$ Some studies reviewed referral letters, ${ }^{67}$ but these are unlikely to carry full information because of the context in which the letters are written, at a time when rapid admission to hospital and initiation of antibiotic treatment are more urgent than the comprehensive recording of symptoms and signs.

We explored how general practitioners made diagnostic and management decisions in children later found to have meningococcal disease. We used a qualitative approach to allow for in-depth exploration of the clinical and contextual features that general practitioners considered important and how this information was used in decision making.

\section{Subjects and methods}

We identified 83 cases of meningococcal disease in children and teenagers under 16 years old that occurred in two hospitals in South Glamorgan from January 1994 to December 1996 using hospital morbidity data. Cases without positive results on culture of blood or cerebrospinal fluid were included when a meningococcal disease had been diagnosed in hospital on the basis of the clinical presentation and course of the illness and when complete antibiotic treatment was given. We found hospital notes for 63 cases $(76 \%)$, and we analysed general practitioner referral letters, admission clinical notes, and bacteriological data. Thirty one children were selected by purposive sampling so that the cases selected would represent the full range of presentations, management, and outcome. These children were referred by 26 general practitioners, who were interviewed using semistructured audiotaped interviews. 
All interviews were conducted by one of the authors (SG). The main part of the interview focused on the general practitioner's previous experiences of the disease, a description of the particular case, and the key features of the case that he or she had considered important in the management. These questions were refined during the pilot and study interviews. Interviews were transcribed word for word; in two cases, notes were taken: in one because the general practitioner preferred not to be recorded and in the other because the equipment failed. Copies of all transcripts were sent to the participants for clarification. Clinical information given by general practitioners was also verified by examination of referral letters and hospital notes. The data were assembled on a matrix using Word for Windows Version 6.0a to allow for comparison of categories for each interview. Patterns and differences were explored. The process was iterative, in that later interviews probed new emerging themes. Another of us (PO) helped validation by independently reading transcripts and listening to all interviews.

\section{Results}

All 26 general practitioners contacted agreed to participate. Their mean age was 42 years (range 29-55 years), and 10 were women. These data were similar to those for all general practitioners in South Glamorgan. Twenty four were principals in general practice, with a mean duration of 11 years' service. Fifteen were members of the Royal College of General Practitioners and 18 had undergone some postgraduate training in paediatrics. The mean interval between case and interview was 61 (SD 39) weeks. Nine general practitioners commented independently on how clearly the events remained in their minds, and the detailed descriptions of 10 others implied good recall. Four general practitioners had some difficulty in describing certain details, and three did not remember the cases very well, but they were able to refer to their clinical notes.

Our subsample of 31 children was similar to the total of 63 children admitted with meningococcal disease with regard to age and sex distribution, the number of times seen by a general practitioner before admission, the duration of illness before admission, the proportion with a rash on admission, and outcome (table).

\section{Rashes}

Non-blanching rash-Of the 31 children, 16 presented with haemorrhagic rashes. The presence of a non-blanching rash was the most important factor in deciding whether to admit these children. "He had an obvious purpuric rash starting on his legs and trunk, and it was clear what the diagnosis was ... It's the rash you see in books" (general practitioner 1 ).

Other explanations-A purpuric rash influenced decisions to admit, but it also led to diagnostic difficulties. Henoch-Schönlein purpura was considered to be a more likely diagnosis in four children because the rash was present only on the legs, and one child without a fever was thought to have thrombocytopenic purpura.

Extent and distribution of the rash-In eight children the haemorrhagic rash was localised, most commonly on the legs or buttocks. In some cases it consisted of
Comparison of general practice sample of 31 children with all 63 children admitted with meningococcal disease. Values are numbers (percentages) of patients

\begin{tabular}{|c|c|c|}
\hline & $\begin{array}{c}\text { General practice } \\
\text { sample }(n=31)\end{array}$ & $\begin{array}{c}\text { Hospital patients } \\
(\mathrm{n}=63)\end{array}$ \\
\hline \multicolumn{3}{|l|}{ Age group: } \\
\hline $0-12$ months & $7(23)$ & $20(32)$ \\
\hline 13-24 months & $5(16)$ & $6(10)$ \\
\hline 24 months-5 years & $10(32)$ & $20(32)$ \\
\hline$>5$ years & $9(2)$ & $17(27)$ \\
\hline \multicolumn{3}{|l|}{ Duration of illness (hours): } \\
\hline $0-24$ & $23(74)$ & $44(70)$ \\
\hline $25-48$ & $2(6)$ & $11(18)$ \\
\hline$>48$ & $6(19)$ & $7(11)$ \\
\hline Missing data & 0 & $1(2)$ \\
\hline \multicolumn{3}{|c|}{ No of contacts with GP during present illness (within 1 week): } \\
\hline None & 0 & $4(6)$ \\
\hline 1 & $19(61)$ & $33(52)$ \\
\hline 2 & $10(32)$ & $17(27)$ \\
\hline $3-5$ & $2(7)$ & $2(3)$ \\
\hline Missing data & 0 & $7(11)$ \\
\hline \multicolumn{3}{|l|}{ Outcome: } \\
\hline Full recovery & $26(84)$ & $52(83)$ \\
\hline Neurological sequelae & $2(7)$ & $2(3)$ \\
\hline Physical sequelae & 0 & $2(3)$ \\
\hline Death & $2(7)$ & $4(6)$ \\
\hline Still to be followed up & $1(3)$ & $2(3)$ \\
\hline Missing data & 0 & $1(2)$ \\
\hline
\end{tabular}

only one or a few spots. "I remember ... examining him reasonably carefully, seeing a spot on his tummy ... and prodding it with my finger ... thinking it was a freckle. And I noticed it didn't blanch" (general practitioner 2).

Non-petechial rashes-Six children had blanching macular rashes. In four of these the doctors suspected the rash might be a marker of serious illness. Three were described as consisting of small red lesions, $1-2 \mathrm{~mm}$ in diameter, which blanched with pressure.

\section{Abnormal illness}

Twenty two children had clinical features not normally expected in children with acute self limiting febrile illnesses. Nine children were abnormally lethargic. Five were described as almost motionless and not wanting to be moved, while three children seemed to be vacant and did not make eye contact or interact with their parents or the general practitioner. Five children had altered mental states: two were confused, two were behaving abnormally, and one was comatose. Three children had abnormal cries (either high or low pitched), one was crying inconsolably, five were pale, and three were cyanosed. "He was acting strangely, and Mum had said he had become aggressive, which was totally out of character for him" (general practitioner 3).

\section{Puzzling findings}

Unusual or puzzling findings were described in nine children. Two children were feverish but pale and with poor blood circulation. "Thinking about it, he was paler than he should have been for the temperature. That is the one thing that has stuck with me. He was boiling hot, but he wasn't red" (general practitioner 2).

Two children were described as crying when handled, preferring not to be held. Four general practitioners reported being puzzled about children with joint pain, which led them to consider explanations for 
the illness other than acute self limiting causes. "He had a viral rash, macular rash, blanched on pressure, a bit sleepy, a bit irritable and complaining of pain in his knees and ankles, which was a bit odd" (general practitioner 4).

\section{Misleading information}

An apparent focal cause for a febrile illness was sometimes misleading-for example, infected tonsils in one child. In five cases an apparently well looking child was potentially misleading, in that most general practitioners expected children with the rash of meningococcal disease to be systemically unwell. "She was sat in that easy chair looking really, as I say, quite well. Not much different to how you look, frankly" (general practitioner 5).

\section{Role of parents}

Contextual information played an important part in the management decision in 15 cases. Most commonly, this entailed taking into account the context of the family and the perceived degree of parental anxiety, especially when the parents and their consulting patterns were well known to the general practitioner. This was of particular importance in eight of the 15 children who did not present with a non-blanching rash. "So she is a sensible mum-you know, comes along with appropriate problems-so it's nice to have that background. And she came with [her son] who had been irritable, off his feeds, and had vomited twice that morning and she burst into tears ... So that was a major factor in my assessment of the child, I think" (general practitioner 6). "It's one I remember reasonably well and I suppose their family do stick out in your mind because I think she's got three young boys and it's fairly unique in that we would never have done a house call" (general practitioner 7).

In two consultations parents played a part in prompting general practitioners to change their management plans. "Then Dad said he had noticed me prodding [a small petechial lesion] and said: 'What is that? I've not noticed that before.' And that was when I looked again" (general practitioner 2).

\section{Management in primary care}

Thirteen children were given intramuscular penicillin by their general practitioners before hospital admission. None received intravenous antibiotics. Certainty of meningococcal disease and the presence and type of haemorrhagic rash (no rash, localised rash, or widespread rash) were the most important predictors of the use of penicillin $\left(\chi^{2}\right.$ for linear trend $=3.6$ for certainty $(\mathrm{df}=1, \mathrm{P}<0.01)$ and 4.5 for $\operatorname{rash}(\mathrm{df}=1$, $\mathrm{P}<0.05)$ ). Penicillin was given in nine out of 13 cases in which general practitioners were completely certain of the diagnosis and in four out of nine cases in which they were moderately certain. All four children without haemorrhagic rashes who were given penicillin were considered to be very ill. Of these, two had macular rashes, which caused the general practitioners to suspect meningococcal disease, and two had evidence of meningeal irritation. "And so there I was with this extremely sick boy and I didn’t, I really didn’t have a clue what was wrong with him and the only thing that made me really seriously think about meningitis was the fact he'd had this ache in his neck the night before and that he'd become extremely ill quickly.... As meningitis was the only thing I could do anything about I gave him some penicillin" (general practitioner 7).

Neither paediatric training nor experience of meningococcal disease was associated with treatment, although penicillin was given in only one of the five cases in which general practitioners had no previous experience of meningococcal disease.

\section{Discussion}

The clinical information used in decision making by this sample of general practitioners was different from the clinical data described in studies of the presentation at the time of hospital admission. ${ }^{1-6}$ Our qualitative interviews with general practitioners have shown that each child with meningococcal disease presented with a unique and often widely varying combination of clinical symptoms and contextual features. Analysis of these interviews has identified five common clinical rules and techniques that helped general practitioners process each unique dataset.

\section{Clinical features}

Firstly, we have confirmed the key clinical importance of a haemorrhagic rash in diagnosing meningococcal disease in children in primary care. A non-blanching rash was the most important independent factor leading to hospital admission and initiation of antibiotic treatment beforehand. This supports the findings of two previous studies. ${ }^{7-10}$ Haemorrhagic rashes were found in just over half of the children, compared with around $70 \%$ of children under the age of 15 years in two hospital studies. ${ }^{3-7}$ This difference may be accounted for by the rapid onset of rashes between assessment in primary care and assessment in hospital.

Secondly, whereas some children with rashes of meningococcal disease seemed to be well, a rash could have been missed if it was not specifically looked for in ill children. If a haemorrhagic rash is present general practitioners should not be deterred from diagnosing meningococcal disease when the child is otherwise well or when the distribution of the rash is unusual or scanty. ${ }^{15}$ Our interviews show that meningococcal disease may present with a maculopapular rash.

Thirdly, general practitioners were aware of abnormal illness in children and detected features that did not apply to self limiting illnesses. Other children presented with features that were not dissimilar to those of self limiting illnesses, but they had unusual, odd, or puzzling findings which caused the general practitioner to consider a more serious cause for their illnesses.

Fourthly, awareness of parents' help-seeking behaviour played an important part in decision making. Responding to parents' impressions of their ill children was particularly helpful when the family was well known to the general practitioner.

Finally, general practitioners were prepared to discount certain potentially misleading information. For example, they were not deterred by a lack of parental concern or the child being otherwise well when undertaking an appropriately thorough assessment. 


\section{Implications for treatment}

Our findings show that the recommendation that general practitioners start antibiotic treatment ${ }^{16}{ }^{17}$ is largely being followed when the diagnosis is certain. Failure to start treatment seems to be related to uncertainty about the diagnosis, partly because of the tendency to focus on extreme signs. It may be appropriate for general practitioners to use parenteral penicillin more readily in children requiring admission to hospital.

Poor response rates to postal questionnaires have been linked to a lack of perceived relevance of the research to general practitioners. ${ }^{18}$ Our $100 \%$ response rate may indicate that general practitioners consider the diagnosis of meningococcal disease to be an important topic for research, as well as reflecting the acceptability of the method we used to collect data-namely, semistructured interviews with a fellow clinician. Our complex data would probably not have been obtained by a structured questionnaire. Many of the general practitioners welcomed the opportunity to discuss the events leading to admission. As this was a retrospective study, they could have reconstructed their experiences in keeping with management decisions and the outcome or portrayed their clinical decisions in a more favourable manner. However, some of them commented on how clearly the events remained in their minds.

We thank the general practitioners who gave their time to be interviewed for this study. We also thank Professor D P Davies and Drs R Verrier-Jones and P White for their constructive comments and advice, and Mrs H Sottero and Mrs G Jones for their help.

Contributors: SG initiated the primary study hypothesis, undertook the literature search, discussed core ideas, designed the study protocol, audited hospital records, conducted all qualitative interviews with the general practitioners, and participated in analysing data and writing the manuscript. PO had the original idea for the study, discussed core ideas, read the background literature, contributed to the study hypothesis and protocol, audited hospital records, and participated in analysing data and writing the manuscript. RP discussed core ideas, contributed to the design of the study protocol, and participated in analysing and interpreting data and in writing the manuscript. LJ discussed core ideas and contributed to the study hypothesis, design of the protocol, piloting, interpreting data, and writing and editing the manuscript. Christopher Butler and Nigel Stott discussed core ideas and contributed to the study protocol and editing of the manuscript. SG and PO are guarantors of the study.

Funding: None.

Conflict of interest: None.

1 Olcen P, Barr J, Kjellander J. Meningitis and bacteraemia due to Neiserria meningitidis: clinical and laboratory findings in 69 cases from Orebo county, 1965 to 1977. Scand J Infect Dis 1979;11:111-9.

2 Donald P, Burger P, van Zyl L. Meningococcal disease at Tygerburg Hospital. S Afr Med J 1980;60:271-5.

\section{Key messages}

- In primary care the main emphasis in managing meningococcal disease in children is early recognition and initiation of antibiotic treatment

- In this study of 26 general practitioners admitting 31 children with meningococcal disease, a haemorrhagic rash was the most important single factor leading to admission but was present in only half of the children

- When a haemorrhagic rash is present general practitioners should not be deterred from diagnosing meningococcal disease and starting antibiotic treatment if the child is otherwise well or if the rash is scanty or has an unusual distribution

- The general practitioners noted abnormal illnesses with features different from those of acute self limiting illnesses, including unwillingness to interact or make eye contact, altered mental states, and pallor with a high temperature

- Knowledge of parents and their help-seeking behaviour were important in making management decisions

- General practitioners were prepared to discount potentially misleading information

3 Palmer S, Corson J, Hall R, Payne S, Ludlow J, Deere B, et al. Meningococcal disease in Wales: clinical features, outcome and public health management. J Infect 1992;25:321-8.

4 Voss L, Lennon D, Sinclair J. The clinical features of paediatric meningococcal disease, Auckland, 1985-87. NZ Med J 1989;102:243-5.

5 Wong V, Hitchcock W, Mason W. Meningococcal infections in children: a review of 100 cases. Pediatr Infect Dis J 1989;8:224-7.

6 Nielson B, Sorensen HT, Nielson JO. Children admitted for observation of suspected meningitis. Scand J Primary Health Care 1988;6:229-32.

7 Woodward C, Jessop E, Wale M. Early management of meningococcal disease. Commun Dis Rep CDR Rev 1995;5(9):R135-7.

8 Riordan F, Thomson A, Sills J, Hart CE. Who spots the spots? Diagnosis of early meningococcal disease in children. BMJ 1996;313:1255-6.

9 Strang PR, Pugh EJ. Meningococcal infections: reducing the case fatality rate by giving penicillin before admission to hospital. BMJ 1992;305:141-3.

10 Cartwright K, Reilly S, White D, Stuart J. Early treatment with parenteral penicillin in meningococcal disease. BMJ 1992;305:143-7.

11 Cartwright K, Strang J, Gossain S, Begg N. Early treatment of meningococcal disease. $B M J$ 1992;305:774

12 Sorensen HT, Moller-Petersen K, Krarup HB, Pedersen H, Hansen H, Hamburger H. Early treatment of meningococcal disease. BMJ 1992;305:774

13 Kilpi T, Antilla M, Kallio M, Peltola H. Severity of childhood bacterial meningitis and duration of illness before diagnosis. Lancet 1991;338:406-9.

14 Koorevaar R, Bruinzeels M, can der Wouden C, van der Velden K, van Suijlekom-Smit L. Patients with suspected meningitis: a study in general practice. Eur J Gen Pract 1995;1:21-4.

15 Marzouk O, Thomson APJ, Sills JA, Hart CA, Harris F. Features and outcome in meningococcal disease presenting with maculopapular rash. Arch Dis Child 1991;66:486-7.

16 Department of Health and Social Security. Meningococcal infection:meningitis and septicaemia. London: $\mathrm{HMSO}, 1988 .(\mathrm{CMO}(88) 2$.

17 Cartwright K, Strang J, Reilly S, White D. Mortality in meningococcal disease. BMJ 1992;304:116.

18 McAvoy B, Kaner E. General practice postal surveys: a questionnaire too far. BMJ 1989;313:732-3.

(Accepted 28 October 1997)

Fifty years ago

The new NHS: A word to the consultants

To our consultant colleagues, I would say it may be true that the battle ground is in the field of general practice. To some extent that is inevitable. But it is also true that the freedom of one section of the profession is of paramount importance to the freedom of the whole. We say to our consultant friends, "We need a united profession, a profession in which all its elements, whether consultant or general practitioner, whether directly affected or not, acknowledge their duty in this regard to the public and to the future of the profession to which they all belong." The members of the profession in other countries, especially in the Dominions, are watching events in this country with anxious interest. It is our duty and our opportunity by standing firm to secure that this thing does not come into operation in its present form. (Dr Charles Hill's address, 10 January 1948, p 65. See also editorial by Gordon Macpherson, 3 January 1998, p 6.) 